\title{
Comprehensive cancer control in the US: 20 years of progress
}

\author{
Leslie S. Given ${ }^{1} \cdot$ Rachel Coughlin $^{2} \cdot$ Polly Hager $^{3} \cdot$ Kerri Lopez $^{4} \cdot$ Gail Merriam $^{5} \cdot$ Krystal Morwood $^{6} \cdot$ Debi Nelson $^{7}$. \\ Ava Crawford ${ }^{7}$
}

Published online: 22 November 2018

(c) Springer Nature Switzerland AG 2018

This year, 2018, marks the 20th year that the Centers for Disease Control and Prevention (CDC) has supported the United States National Comprehensive Cancer Control Program (NCCCP). The NCCCP emerged from a movement called comprehensive cancer control (CCC), which began as a concept in 1994 and is focused on addressing cancer in a comprehensive manner, across the continuum of cancer control rather than focusing in on one cancer site (e.g., colorectal) or only on one aspect of care delivery (e.g., prevention). CCC is an approach that brings together multi-sector partners to collectively address the cancer burden in a community by leveraging existing resources and identifying and addressing cancer-related issues and needs. In 1999, the Comprehensive Cancer Control National Partnership (CCCNP) was officially formed, bringing together key federal and national cancer stakeholders to support the NCCCP and their CCC coalitions as they develop and implement CCC plans.

This is the third Special Issue on CCC, all published in Cancer Causes and Control. In this Special Issue, we offer reflections on the progress made over the past 20 years in addressing the US cancer burden through the CCC approach and present a range of topics, from a description of how CCC programs and coalitions have evolved over the years to analyses of how they can affect change through policy,

Leslie S. Given

leslie@shconcepts.com

Strategic Health Concepts, Earlysville, USA

2 Texas Department of State Health Services, Austin, USA

3 Michigan Department of Health and Human Services, Lansing, USA

4 Northwest Portland Area Indian Health Board, Portland, USA

5 Massachusetts Department of Public Health, Boston, USA

6 Colorado Department of Public Health and Environment, Denver, USA

7 North Carolina Department of Health and Human Services, Raleigh, USA systems, and environmental approaches in areas such as liver cancer prevention, lung cancer screening, and addressing cancer survivors' wellness. The Special Issue also includes national perspectives and outcomes from the NCCCP and the CCCNP, in their collaborative efforts to "conquer cancer."

In this preface, we highlight both the evolution and progress of the first six NCCCP's funded through CDC. In 1998, CDC and its partners were still working to define what CCC meant, how it could be useful in addressing the burden of cancer in a state, tribe, or territory, and were often more focused on traditional public health issues like education about cancer prevention and encouraging cancer screening. Now CCC programs and coalitions have a broader and evolving role beyond prevention and early detection into cancer diagnosis, treatment, and survivorship issues. CCC coalition partners represent more sectors of the community, are more diverse, and leverage partner strengths in innovative ways to maximize collective efforts. And, CCC plans and implementation of those plans focuses more on integrating advances in cancer control science and research, as well as a focus on policy, systems, and environmental approaches to ensure longer-term change.

\section{Twenty years of progress: examples from the first National Comprehensive Cancer Control Programs and coalitions}

\section{Colorado}

The Colorado Cancer Coalition, the Colorado Cancer Plan, and Colorado's Comprehensive Cancer Control Program have guided the prevention, treatment, and control of cancer statewide for more than 20 years. The Coalition formed in 1993, the first state cancer plan was unveiled in 1996, and Colorado began participating in the NCCCP funded by the CDC in 1998. The long-standing partnership among these 
efforts solidified the fundamental principles of collaboration, coordination, and leveraging of resources.

The current vision and mission of the Colorado Cancer Coalition (http://coloradocancercoalition.org/) is to "Eliminate the cancer burden in Colorado," and "To engage, facilitate, expand and strengthen the statewide network dedicated to improving Colorado lives touched by cancer." The goal of the Coalition is to prioritize efforts and implement strategies in the Colorado Cancer Plan (https://www.colorado. gov/pacific/cancerplan/colorado-cancer-plan). Over the years, the mission and vision language has varied, but the overarching direction to reduce the burden of cancer in Colorado using strategies from the Colorado Cancer Plan has remained.

Each version of the state cancer plan has challenged Colorado's cancer community to think strategically about new partnerships and collective efforts to expand, reach, and improve outcomes. The process for developing the state's cancer plan has continuously relied on contributions from individuals and organizations across Colorado, and has received support from the CDC's NCCCP. The expertise and commitment of state and local health agencies, health systems, and community-based organizations is critical for achieving successful implementation of the state cancer plan.

Since 1996, there have been four iterations of the Colorado Cancer Plan. The initial versions focused on specific cancer types (e.g., lung, breast, cervical, colorectal) and strategies to address them. The current plan addresses strategies via a continuum of care approach, addressing interventions across the areas of prevention, screening and early detection, diagnosis and treatment, and survivorship and palliative care. While there are still strategies specific to cancer types, as in previous versions, partners have realized that many strategies can improve outcomes for multiple cancer types (e.g., implementing cancer screening policies within health systems can improve breast, cervical, colorectal, and lung cancer screening rates). The current plan's structure addresses the need to speak to a broad potential audience such as individuals, health systems, policy makers, employers, and others who are best positioned to implement various strategies.

The Colorado Cancer Coalition's leadership and advocacy for cancer control efforts, together with the Colorado Comprehensive Cancer Control Program's alignment with prevention strategies included in other state chronic disease control programs and initiatives, created synergy and contributed to multiple cancer-related successes from increased funding to improved cancer survival rates.

Established in 2010, the Colorado Breast and Reproductive Cancer Fund provided a mechanism for taxpayers to voluntarily contribute money to support the coalition in implementing its mission and vision. Altered to the Colorado
Cancer Fund in 2012 to be more inclusive, it continues as an important resource for the sustainability of the Colorado Cancer Coalition and provides the opportunity to fund dozens of organizations to implement Colorado Cancer Plan strategies.

One of the most significant initiatives to impact cancer prevention and control in Colorado in the last 20 years was a tax increase on cigarettes and other tobacco products. In 2004 , concerned citizens initiated and voters approved a 64-cent increase on a package of cigarettes and an additional assessment of $20 \%$ on the wholesale price of other tobacco products. The revenue was designated for increased provision of health care services and tobacco prevention education and control efforts. This increase temporarily brought Colorado's tobacco tax rate among the highest in the nation, although other states have since passed their own increases and Colorado now ranks 35th in the price of tobacco products. Each year, $32 \%$ of revenue (or roughly $\$ 35$ million $^{1}$ ) from this measure is legislatively allocated to four programs administered by the Colorado Department of Public Health and Environment. Each program has responsibility for implementing some of Colorado's Cancer Plan strategies.

1. Cancer, Cardiovascular Disease and Pulmonary Disease (CCPD) Competitive Grants Program (https://www. colorado.gov/pacific/cdphe/A35-ccpd-grants).

2. Tobacco Education, Prevention and Cessation Grant Program (https://www.colorado.gov/pacific/cdphe/A35tobacco).

3. Women's Wellness Connection (https://www.colorado. gov/pacific/cdphe/womens-wellness-connection).

4. Health Disparities Grant Program (https://www.color ado.gov/pacific/cdphe/categories/services-and-infor mation/health-equity).

These programs award money to projects and organizations that implement chronic disease prevention and control strategies and the majority of these efforts align with numerous parts of the Colorado Cancer Plan.

The rapidly changing science of cancer has shaped Colorado's efforts as well. As the American Cancer Society (ACS) reports, "Scientists have learned more about cancer in the last 2 decades than had been learned in all the centuries preceding" [1]. Cancer screening and treatment is becoming more complex, personalized, and effective. 5 -year survival rates for cancer are increasing, and this, along with Colorado's status as a relatively healthy state, contributes to a somewhat unique challenge of having a high number of cancer survivors. The Colorado Central

\footnotetext{
$\overline{1}$ This number is annually variable and generally decreases from the prior year due to a consistent decline in tobacco sales and use.
} 
Cancer Registry estimates that approximately 258,000 Coloradans are living with active cancer or a history of cancer, and predicts that this number will grow to 325,000 by 2025 . Cancer survivorship issues comprise an important component of Colorado's cancer control strategy because of the Coalition's recognition that this population is at increased risk for cancer, other chronic diseases, and many other health issues.

In alignment with the Affordable Care Act (ACA), Colorado took advantage of the opportunity to expand Medicaid. These efforts allowed Colorado's rate of uninsurance to go from $15.8 \%$ in 2011 to $6.5 \%$ in 2017 , as reported in "Colorado's New Normal: State Maintains Historic Insurance Gains." (https://www.coloradohealthinstitute.org/sites/defau 1t/files/file_attachments/2017\%20CHAS\%20DESIGN\%20 FINAL $\% 20$ for $\% 20$ Web.pdf). Insurance coverage opened opportunities for state preventive screening programs to reduce the funding directed to health care services and increase focus on more sustainable health systems changes. However, only modest gains in overall state cancer screening rates have been reported since ACA adoption, so more needs to be done at the systems and individual levels to increase the utilization of preventive screening coverage available.

Although the reasons for improving cancer outcomes over the years are multifaceted, efforts guided by the state's Comprehensive Cancer Control Program, Colorado Cancer Coalition, Colorado Cancer Plan, and the strategic alignments with related efforts have played an important role in this progress. Cancer death rates declined from $170.9 \%$ in 1999 to $136.0 \%$ in 2016 . In the same 18 -year period, breast cancer death rates declined by $3.3 \%$, prostate cancer death rates declined by $7.8 \%$, colorectal cancer death rates declined by $6.4 \%$, and lung cancer death rates declined by $13.0 \%$. In 1998 , for all cancers combined, the 5-year survival rate was $63.1 \%$. For patients diagnosed between 2008 and 2012, the 5-year survival rate is $67.2 \%$ [2].

Despite the substantial gains in cancer prevention and control over the last 20 years, new and recurring challenges in the area of cancer control demonstrate there is more work to be done. Some of the highest priority initiatives for the state moving forward include:

- Further tobacco use reduction, including increasing the price point of tobacco products and continuing to implement evidence-based efforts to reduce tobacco use initiation and increase tobacco cessation.

- Enhanced health systems approaches to increase use of preventive services, including cancer screening and cancer prevention vaccinations.

- Enhanced survivorship initiatives, including the use of treatment summaries and care planning, health navigation and connecting to chronic disease prevention and management initiatives.
- Increased focus on eliminating disparities in cancer outcomes including health navigation, linkages to resources, and access to care.

- Enhanced efforts to connect with other chronic disease prevention and management initiatives to allow for focus on whole-person health.

\section{Massachusetts}

When the Massachusetts Department of Public Health initiated a Comprehensive Cancer Prevention and Control Program 20 years ago, there were a number of significant funding and programmatic differences from today's current efforts in the state. 20 years ago, the passage of a significant cigarette tax passed in 1992 continued to generate funding for a robust and in-depth tobacco program ensuring a dedicated approach to tobacco education including largescale media campaigns. Therefore, Massachusetts's cancer prevention and control program staff and partners primarily collaborated with the Tobacco Program in its advocacy for prevention and policy changes related to tobacco.

In 1998, the Massachusetts Comprehensive Cancer Prevention and Control Program also benefited from dedicated state funding for cancer separate from the initial CDC NCCCP funding (e.g., both prostate, breast, cervical, and colorectal) which supported large-scale projects and spurred partner interest in joining the state Comprehensive Cancer Coalition. This led to a number of academic partners directly contributing staff time and participating in the coalition. The coalition consisted of a wide array of clinicians and administrators from institutions such as Boston Medical Center, Massachusetts General Hospital, and Dana Farber Cancer Institute.

Due to the extensive involvement of academic and research health care experts, the coalition was primarily Boston centric. Over time the coalition expanded to be more statewide and to represent a broader, more diverse array of partners. This was in part due to increasing cancer disparities that existed, despite high screening rates and advanced treatment options in the state. Subsequently starting in 2012 and extending to 2018, the Massachusetts Cancer State Plan has prioritized cancer disparities, which at one point led to a separate Cancer Disparities Work Group within the coalition.

Throughout the past 20 years, ACS has served as a key partner by providing the Massachusetts Comprehensive Prevention and Control Cancer program with significant in-kind support such as staff time, meeting space, and food or partner outreach thereby supporting a number of critical comprehensive cancer initiatives that would not have occurred without ACS's involvement. However, now ACS is no longer the primary organization doing cancer-related walks and cancer-related fund raising. Instead there has been 
a dramatic rise in number of cancer centers conducting their own fundraising specific for cancer including walks with all of the money raised being channeled into individual hospital cancer inpatient and outpatient programs.

By the late 1990s, there was some staff turnover at Program Director level within the Comprehensive Cancer Program at the Department of Public Health (DPH) which impacted communication and collaboration with coalition members. It was during this time that the Comprehensive Cancer Coalition considered becoming a $501 \mathrm{c} 3$ to potentially receive separate funding but did not pursue it when they realized it would be competing against DPH.

In 2008, a new Commissioner at DPH felt the need to restructure the Comprehensive Cancer Coalition to be more effective and impactful by creating an Advisory Committee that could provide input and guidance more readily than a large coalition. The Comprehensive Cancer Program recruited several experienced partners and engaged in strategic planning to ensure the Advisory Committee would function well and provide the needed input. Then in 2014, the Massachusetts Comprehensive Cancer Program created a three-tier structure of organizational functioning and changed its name to its current one: Massachusetts Comprehensive Cancer Prevention and Control Network.

One of the greatest benefits of CCC has been the focus on convening partners. The "power of partnerships" became a clear and resounding reality for the Massachusetts Comprehensive Cancer program. The impact of the unique and dynamic process of bringing together a diverse group of clinicians, researchers, survivors, and advocates to collaborate has contributed to a decrease in the cancer burden in Massachusetts by, for example, achieving some of the highest screening rates in the country along with improved survival of those diagnosed with cancer.

During the late 1990s and early 2000s, the meeting room for the original coalition would be overflowing with members. Partners were actively involved in writing grants and leading and facilitating Work Groups. There were a number of reasons for strong and vibrant partner participation. These included the following: (1) partners wanted to be involved in designing and implementing innovative projects; (2) partners wanted to contribute to decisions made on policy; (3) partners wanted to be sure that their individual or organizations' perspective was represented; (4) partners saw and experienced a true sense of a value-added benefit for members through effective networking and collective impact; (5) partners felt that their voices were heard in the mission of the cancer coalition; and (6) partners could see how the results of collaboration directly benefited their organization, such as the development of a Survivorship Wellness Guide and the initiation of a Lung Cancer Screening Learning Collaborative, to name a few.
As one long-term coalition member, Janet McGrail Spillane then Vice President for ACS said, "We were lucky enough to have a group of people come together and write the first state cancer plan. It was really important that the plan was developed by a group of different representatives who were willing to roll up their selves and contribute. I always felt as though it was true public health in action where small ideas translated into larger impact for cancer."

Here are some areas of success for the Massachusetts Cancer Control program, including our recent new cancer state plan: ftp://ftp.cdc.gov/pub/Publications/Cancer/ccc/ massachusetts_ccc_plan-508.pdf.

1. Survivorship: In addition to implementing a wellregarded Survivorship State Summit in 2008, the Survivorship Work Group directed a statewide survey on treatment summaries and care plans and developed an innovative Massachusetts Cancer Survivorship Wellness Guide: https://www.mass.gov/wellness-guide-for-cance r-survivors.

2. Prostate: Developed a Prostate Cancer Shared Decision Making Aid and Fact Sheet: https://www.mass.gov/servi ce-details/prostate-cancer-resources and two Shared Decision Making videos, one for average risk men: https ://youtu.be/XICtKN4qDik and one for high-risk men: https://youtu.be/sVD1yGd0mqA.

3. Colorectal: Oversaw production of small media campaign targeting Black and Hispanic men and their family members and a lower literacy colorectal cancer education booklet: https://massclearinghouse.ehs.state.ma.us/ images/thumbs/CA1373.jpg.

4. Palliative care: Conducted a statewide survey on availability of palliative care and facilitated regional forums in each area of the state leading to several regional collaborative groups.

5. Lung cancer: Implemented a statewide survey of LowDose CT sites offering Lung Cancer Screening. The results were used to launch the Massachusetts Lung Cancer Screening Learning Collaborative.

\section{Michigan}

The Michigan Cancer Consortium (MCC) was formed in 1987 as an advisory body to the state health department. Key accomplishments over the succeeding 10 years include mammography quality, establishment of a breast and cervical cancer program, and a prostate cancer consensus conference [3]. In 1997, the MCC restructured to become an organization of organizations with 31 founding members. By 2000 , the MCC had selected ten cancer control priorities for statewide implementation, developed a strategic plan, and solidified its infrastructure [4]. 
The initial ten priorities selected by the MCC in 1998 were comprehensive. The priorities addressed high burden cancers with public health interventions, and looked at cancer issues from prevention through end of life care. Though priorities have changed a few times via selection processes involving the MCC and its board of directors, the priorities in place today continue to be comprehensive. The current cancer plan goals and priorities are organized by prevention, early detection, diagnosis and treatment, and quality of life [5].

The Cancer Plan for Michigan, 2016-2020, is being implemented by nearly 100 organizational members from across the state. The interventions in the plan are evidencebased and include targets to measure improvement [5]. Progress is measured online using a Cancer Plan Dashboard [6] (see the dashboard in the Evolution of Plans and Partnerships article in this special issue). Some priorities, increasing cancer clinical trial enrollment and colorectal cancer screening, are still relevant 20 years later while others are products of emerging science and trends, such as human papilloma virus (HPV) vaccination and survivorship care plans.

With over 20 years of work as a statewide coalition, the MCC has had numerous achievements to celebrate. Early on in 2001, an important reason for celebration was an accomplishment addressing cancer clinical trials. The product was a statewide consensus agreement among cancer care providers, payers, advocates, and policy makers. The voluntary agreement addressed third-party payer coverage of patient care costs in an effort to improve access to clinical trials, among other important issues. The Michigan Working Group to Improve Cancer Outcomes was responsible for negotiating the agreement. 15 years later, the MCC worked on a new clinical trials priority, specifically to increase the percentage of adults participating in cancer treatment trials from 4.4 to $4.8 \%$. The achievement of that priority was marked in 2017, based on data from the previous year [4].

Collaboration has been key to addressing tobacco control including passage of the Dr. Ron Davis Smoke-free Air Law that went into effect in May 2010 [4]. Following implementation of the law, secondhand smoke biomarkers including NNAL, which is a tobacco-specific lung carcinogen, were significantly lower and reported respiratory health symptoms improved among bar employees in Michigan [7]. Recent collaborative efforts, which began in 2012, target tobacco dependence treatment for cancer survivors. The focus is on changing clinical practice so patients being treated for cancer are identified, advised to quit, and offered tobacco dependence treatment. Cessation services are being provided through the Michigan Tobacco Quitline [8]. Since inception, 4,347 cancer survivors, which include patients in treatment, have enrolled in the quitline for tobacco dependence treatment [9].
As 2017 drew to a close, the MCC Board of Directors selected new priorities to focus its efforts on for 2018-2019 [4]. Three remain the same since 2016. Those include increasing HPV vaccination rates, increasing colorectal cancer screening, and increasing clinical trial enrollment. New to the list is reducing physical pain caused by treatment among cancer survivors. When the timeline for these priorities draws to a close in 2019, the Consortium will have a methodical process underway to update the cancer plan, select new priorities, and begin its implementation efforts afresh as it has for 20 years.

\section{North Carolina}

The North Carolina Comprehensive Cancer Control Program (N.C. CCCP) was established in 1945 by the General Assembly at the urging of the Women's Field Army (now the ACS) and the North Carolina Medical Society. N.C. CCCP was initially funded by a grant from the Society. In 1947, the General Assembly appropriated $\$ 36,700$ for the program. In 1993, North Carolina became one of the original six CDC-funded NCCCP recipients. N.C. CCCP is currently housed in the N. C. Department of Health and Human Services, Division of Public Health, Chronic Disease and Injury Section, Cancer Prevention and Control Branch (Cancer Branch).

North Carolina was one of the four states chosen for the CDC and National Association of Chronic Disease Director's Program Integration Project. During the project period, the N.C. Chronic Disease and Injury Section's workflow and networking were examined. In 2011-2012, it was decided to combine the N.C. Breast and Cervical Cancer Control Program (N.C. BCCCP), N.C. WISEWOMAN Project (N.C. WISEWOMEN), and N.C. CCCP programs into one branch with a single manager.

The N.C. Central Cancer Registry works closely with the Cancer Branch as well. The coordinated work of the three programs and the Registry proves to be beneficial to all in several ways. Sharing of data, program deliverables, project activities, and grant work plans enables all to coordinate and expand the work of the branch. The Leadership Team meets regularly to discuss projects and future ideas to facilitate coordination. The Cancer Branch collaborates with the other branches in the N.C. Chronic Disease and Injury Section to address the risk factors associated with cancer.

The N.C. CCCP uses an integrated approach to guide CCC efforts and promote screenings. N.C. CCCP expands partnerships and community engagement to foster education and training opportunities and to facilitate resource sharing, community, and clinical linkages among partner networks. N.C. CCCP uses a health equity lens to help reduce gaps through partnerships and resource sharing to influence policy and systems change. 
The N.C. CCCP works to ensure a comprehensive and collaborative approach to address the state's cancer burden through A Call to Action: North Carolina State Comprehensive Cancer Control Plan 2014-2020 (N.C. Cancer Plan) (http://www.tinyurl.com/nccancerplan). N.C. CCCP brings together key partners and organizations to implement and evaluate the N.C. Cancer Plan. There are two large partner network groups that are connected through our N.C. Cancer Coalition: the N.C. Advisory Committee on Cancer Coordination and Control (ACCCC) members and partners and the N.C. Colorectal Cancer Roundtable (N.C. CRCRT).

In 1992, the process to create the (ACCCC) began because of a Cervical Cancer Task Force report to the North Carolina General Assembly. This report determined there should be a study commission on cancer and they proposed the establishment of statewide cancer coordinating and control body. In 1993, the N.C. General Assembly mandated the ACCCC be formed. A statue (130A-33.51) was established for 34 designated members to be appointed by the Governor, Senate, and House of Representatives. ACCCC serves as a statewide advisory board in cancer-related legislation, policy, regulations, and standards. It meets bi-annually and annual reports are delivered to the N.C. DHHS Secretary. The ACCCC is mandated to prepare a N.C Cancer Plan for statewide implementation by an interagency comprehensive, coordinated cancer control program (N.C. CCCP). In 1996, the first cancer control plan was presented to the N. C. General Assembly members.

Additionally, the ACCCC creates and updates position statements on cancer screening. The position statements are derived by consensus of the ACCCC members to determine best practices and recommendations for cancer screenings such as the prostate-specific antigen (PSA) test, use of the HPV vaccine, or use of the best or more appropriate cancer screening test versus another. The position statements serve as guidelines for legislatively mandated insurance coverage. Due to the amount of research that is done in establishing the position statement recommendations and the wide representation of the ACCCC, the position statements provide credibility to health care professionals across the state.

The ACCCC is comprised of five subcommittees to implement the cancer plan. Below are just a few success stories from work that has been accomplished.

ACCCC Care and Treatment Subcommittee members collaborate to address the continuum of services from cancer diagnosis to treatment, care, survivorship, and palliative care. They work closely with N.C. CCCP to coordinate an annual statewide Cancer Survivorship Summit. The Summit brings together cancer survivors and caregivers to share their experiences; learn the latest information on cancer treatments, research, and psychosocial issues; learn self-advocacy skills and empowerment tools to enhance the survivors' quality of life. The Summit reaches at least
160 cancer patients and survivors annually. Through strong partnerships and financial support with various cancer centers and professional organizations, scholarships are offered to individuals with financial challenges.

The N.C. Cancer Prevention and Control Branch, ACCCC Evaluation Subcommittee, and other partners released the North Carolina cancer burden document titled, Reducing the Burden of Cancer in North Carolina: A Data and Resource Guide for Communities to Fight Cancer (http://publichealth.nc.gov/chronicdiseaseandinj ury/cancerpreventionandcontrol/docs/ReducingtheBurd enofCancerResourceGuide.pdf, http://tinyurl.com/nccan cerburdendoc) in November 2017, to communicate the N.C. cancer burden status to target prevention and control efforts. This burden document is a companion document to A Call to Action: North Carolina Comprehensive Cancer Control Plan. It provides a mid-cycle update on targets established and updates recommended evidencebased interventions for the six N.C. priority cancers (lung, colorectal, breast, prostate, melanoma skin, and cervical) and data-informed recommendations about where interventions should be targeted. Both documents focus on the six priority cancers. The Cancer Burden Document uses data visualization to provide a one-stop shop of easy-tounderstand data to illustrate where the priority cancers are concentrated the highest, the groups most affected by them, and what state and local partners can do in their communities to address these cancers through evidencebased strategies and resources available. Additionally, it provides current, easy-to-use data for organizations to use for program planning and grant applications to bring in resources to address cancer at the community level.

The ACCCC Prevention Subcommittee includes representation from the N.C. Radon Program. This Subcommittee developed a partnership with N.C. Real Estate Commission to increase awareness of the need to test homes for radon and decrease risk for lung cancer by reducing radon in homes. A handout, Safe at Home: Preventing Lung Cancer by Reducing Radon in the Home, was developed for real estate agents and brokers to use with clients. N.C. Real Estate Commission members and a key ACCCC Prevention Subcommittee member from the N.C. Radon Program developed a continuing education course on radon that included the new handout. All new real estate agents/brokers are receiving the radon training and it is available to current agents/brokers to earn continuing education credits to maintain their license. The handout has been used by other state agencies, local health departments, provider organizations, and other stakeholders. All N.C. county health departments distribute radon test kits and communicate messaging. Currently, the members are in the process of developing a 3-min video about the link between lung cancer and radon, promoting testing the home for radon, and the value in taking action. 
The N.C. CCCP collaborated with the N.C. BCCCP, N.C. WISEWOMAN, the N.C. Community and Clinical Connections for Prevention and Health Branch, N.C. State University, and N.C. Cooperative Extension to develop a chronic disease unit that includes a cancer lesson plan in the Faithful Families Eating Smart and Moving More curriculum http:// www.faithfulfamiliesesmm.org/. Faithful Families is a group education program that promotes healthy eating and physical activity in communities of faith. The Faithful Families curriculum has been accepted as a "Practice-Tested Intervention" by the Center of Excellence for Training and Research Translation at University of North Carolina at Chapel Hill. This curriculum has been adopted by and implemented in 84 North Carolina faith-based organizations and in other states, giving it a national presence. The new cancer lesson plan titled "You Can Reduce Cancer Risk" provides education on specific cancer-related risk factors and strategies that participants can use to help reduce their risk for cancer.

The Cancer Branch, ACS, and Mecklenburg County Health Department partnered together in 2014 to create the N. C. Colorectal Cancer Roundtable (N.C. CRCRT). It began with a Colorectal Cancer Forum in February 2015 to bring interested parties together to begin the process. N.C. CRCRT has a steering committee and its partners are comprised of colorectal cancer professionals and experts. While organized as an independent organization, N.C. CRCRT is supported by the N.C. CCCP and ACCCC, with the Cancer Branch manager serving as the executive director. N.C. CRCRT uses the N.C. Cancer Plan as a guide for addressing colorectal cancer. Members are working on strategies to increase better access to care for screening, promote appropriate screening, and improve screening coverage by insurance companies. In July 2017, N.C. CRCRT was selected to attend the Comprehensive Cancer Control National Partnership's Colorectal Cancer Forum, supported by CDC and ACS. A team of six members attended the forum and created an action plan to develop a CRC screening program with a Federally Qualified Health Center.

In the future, N.C. CCCP plans to build and expand on current successes of program activities and expand partnerships to continue to use the integrated approach to implement the N.C. Cancer Plan. A new strategy starting soon is to create Community Cancer Networks that will bring partners together to address cancer at the community level. The goal will be to identity local champions and key leaders to determine how they want to address the cancer burden in their community. N.C. CCCP staff will play a key role in assisting them to identify local and state resources to implement their plans. This will start as a pilot project with hopes of expanding the model statewide. N.C. Cancer Branch, N.C. CCCP, ACCCC, N.C. CRCRT, and other organizations and partners will continue to work together to reduce barriers to access to care, continue to promote prevention and early detection, work to improve policy and system changes, and improve services and support systems to cancer survivors.

\section{Northwest Portland Area Indian Health Board}

Northwest Portland Area Indian Health Board (NPAIHB) was the first tribal organization to receive CDC NCCCP funding in 1998. The Northwest Tribal Comprehensive Cancer Control Project (NTCCP) was created to form a tribal cancer coalition covering multiple states, develop a tribal comprehensive cancer plan, design a tribal behavioral risk factor survey, and collaborate with a wide network of partners including federal, state, academic, non-profit, and private industry partners.

The Northwest Tribal Cancer Coalition (Coalition) over the years has grown from eight members in 1999 to current membership ranging from 40 to 50 . We have had representation from all of the 43 tribes in the Northwest. The Coalition meetings have progressed over time with feedback from membership to provide a clinical component and trainings on various topics such as HPV, chronic disease, various cancers, cancer screening, tobacco, physical activity, nutrition, healthy cooking, and cancer survivorship. Coalition members have an opportunity to share successes, challenges, and to network on strategies to address cancer. We have also added a resource component at meetings for partner organizations and cancer centers to share their initiatives and resources with the tribal programs.

Early on, NPAIHB's tribal CCC program focused on building partnerships to work with the Coalition. We currently have over 100 partners from various entities inclusive of State and Tribal Breast and Cervical Cancer Programs, Comprehensive Cancer, Cancer Registries, NCI and Community Cancer Centers, academic institutions, advocacy, and supportive care non-profit organizations. The NTCCP, in collaboration with member tribes and the NCI Partnership Program, developed an Evidence-Based curriculum, called Cancer 101 (http://216.243.141.9/programs/project/ ntccp_cancer_101). Working with the NCI-funded Northwest Tribal Cancer Navigator Project, staff developed a journal for newly diagnosed American Indian/Alaska Native (AI/AN) cancer patients, called Appointment Companion. Other groundbreaking tools developed by NTCCP and member tribes include the following: Northwest Tribal Cancer Resource guide, Northwest Tribal Colorectal Cancer Toolkit, regularly updated AI/AN state-specific cancer fact sheets, Oregon Tribal CHANGE Tool Summary Report, Cancer Among Northwest AI/AN, and Northwest AI/AN Mortality Report [10, 11].

In addition, NTCCP has provided annual cancer clinical update training since 1999 to tribal primary care providers including Certified Medical Education and Certified Nurses Education. This training is a partnership with the 
Portland Area Indian Health Service, Oregon Health and Science University, Legacy Cancer Institute, and various academic organizations to cover topics including cancer navigation, tobacco cessation and prevention, HPV immunization, colorectal cancer screening, chronic disease, liver cancer prevention, after care summary for cancer survivors, lung cancer screening, and the complexity of tracing and documenting cancer in the electronic health record. Other training provided by NTCCP includes tobacco policy and cessation, traditional foods, breast feeding, Women's health, HPV immunization, colorectal cancer screening, and a multidisciplinary training with Tribal Epi Center projects. The multidisciplinary training (titled "Risky Business") includes projects addressing diabetes, tobacco, immunizations, targeting and marketing $\mathrm{AI} / \mathrm{AN}$ youth in tobacco advertising, nutrition, and physical activity. NTCCP provides valuable culturally appropriate resources and curriculum to our tribal programs including Clinical and Community Tobacco Cessation, Indian Health Service: My Native Plate, Healthy Lifestyle, Weight Management, Weight Management for Kids, Physical Activity Kit, Let's Move in Indian Country, Tribal Tobacco Policy Toolkit, and Community Health Representative's Colorectal Cancer Screening.

NTCCP continually provides technical assistance and support on cancer issues that the Northwest tribes have identified. We have also worked closely with our data project staff to provide tribal-specific data in addition to state-level $\mathrm{AI} / \mathrm{AN}$ cancer fact sheets.

The Northwest Tribal 20-Year Cancer Plan was solely developed by a tribal and stakeholders work group in 1999. The workgroup included tribal partners and CDC-funded programs from Idaho, Oregon, Washington, as well as the Native American Rehabilitation Association, South Puget Intertribal Planning Agency, Native Wellness Institute, Native American Cancer Research, American Heart Association, ACS, and the National Cancer Institute's Cancer Information Service.

The original Northwest tribal BRFSS project in 2001 was the first regional tribal Behavior Risk Factor Surveillance System (BRFSS) with participation from six Northwest tribes. In the past 5 years, six additional tribal BRFSS surveys were completed. A unique aspect of tribal BRFSS is the development of surveys including standard CDC modules along with each tribe's specific modules. The tribalspecific modules are created by tribal teams to incorporate locally relevant health issues (for example, program needs, cultural issues, homelessness, cultural resiliency, and historical trauma). Other successful projects that had roots in the NTCCP included the Tribal Elder Diet and Nutrition Study, and the tribal data linkage project (correcting for AI/AN racial misclassification with the state cancer registries) [12].

We currently provide support to tribal programs for local implementation of the cancer plan with tribal communities, requiring tribal programs to identify objectives from the 20-Year Northwest Tribal Cancer Plan. Examples of these tribal implementation activities include breast and cervical cancer screening and education events, healthy cooking education, community gardens, community events for men focused on cancer prevention and screening education, Pink Paddle women's cancer survivorship and cancer screening, survivorship groups, and establishing local tribal-specific cancer coalitions.

The most marked change since the NTCCP began is the level of community readiness to address cancer issues. The 2002 President's Cancer Panel (https://deainfo.nci.nih.gov/ advisory/pcp/archive/pcp02rpt/YakamaBook.pdf), hosted by the Yakama Nation, shared issues faced by tribal members in the Northwest.

Many of the challenges to addressing cancer remain, but tribal communities are now actively discussing cancer issues and publicly sharing experiences of the impact of cancer. There is a tangible change in tribal communities' readiness to openly talk about cancer.

The NTCCP will continue to provide and seek support for the following: Policy, systems, and environmental changes, tobacco cessation, survivorship, screening, and navigation. The successes of local tribal cancer action plans will be sustained through support for funding of tribes and their cancer action plans. The Northwest Tribal Cancer Plan will continue to be updated as new data, and relevant information is available. NTCCP continues to work with Northwest tribal clinics to provide quality data back to the community. The challenge of having patient-specific treatment summaries and cancer survivorship plans available to tribal members and tribal clinics will be addressed to increase evidencebased care after completion of treatment.

We are optimistic that working closely with our tribal coalition members, our partners, and our CDC colleagues, we will impact the reduction of cancer incidence and mortality. We strive for improvement in cancer survivorship and quality of life toward the next seven generations of healthier, cancer-free Indian communities.

\section{Texas}

In 1998, the Department of State Health Services (DSHS) received funding from the $\mathrm{CDC}$ to implement the Texas Cancer Plan through its Texas Comprehensive Cancer Control Program (TCCCP). In partnership with the Texas Cancer Council, now known as the Cancer Prevention and Research Institute of Texas (CPRIT), DSHS initiated the formation of the Texas Comprehensive Cancer Control Coalition to facilitate collaboration and communication among a wide range of member agencies working in cancer control. In 2003, by mutual agreement between DSHS and the Texas Cancer Council, active administration of the Coalition transferred to 
the Texas Cancer Council. DSHS continued to provide funding to the Coalition and played an integral role in its success.

In 2006, the DSHS again assumed administration of the Coalition through TCCCP. To commemorate its 10th anniversary in 2009, the Coalition changed its name to the Cancer Alliance of Texas (CAT). TCCCP continues to provide support to CAT and plays an integral role in its continued success. As of 2018, CAT has over 150 members and four priority area work groups: Tobacco Control, Survivorship, Colorectal Cancer Screening, and HPV Vaccination. CAT's mission is to engage organizations, agencies, institutions, and individuals to work collaboratively to reduce the impact of cancer in Texas and promote the Texas Cancer Plan [13]. CAT members have been actively engaged in the development and update of the Texas Cancer Plan, most recently released in 2012, which serves as the blueprint for cancer control activities in Texas.

Priority areas for 2012-2016 were selected by experts in the field of cancer prevention and control and public health and based upon review of cancer trends, health disparities, and available evidence-based strategies, and, if implemented in systematic and comprehensive ways, will have a significant impact on the human and economic cancer burden in Texas. The Plan addresses the entire spectrum of cancer control, from cancer research, prevention, and control areas including risk reduction, early detection, and screening, to diagnosis, treatment, palliation, quality of life, survivorship, research, and commercialization. Identifying the challenges and issues that affect Texas, the Plan presents a set of goals, objectives, and strategic actions to help inform and guide communities and partners in the fight against cancer.

CPRIT is charged with development, promotion, and implementation of the Texas Cancer Plan. During the 80th Legislative Session in 2007, House Bill 14 renamed the Texas Cancer Council as the Cancer Prevention and Research Institute of Texas (CPRIT), and voters later adopted House Joint Resolution 90 to establish CPRIT in the state constitution [14]. CPRIT was created to develop the Plan, fund groundbreaking cancer research, and prevention programs and services in Texas, with the goal to expedite innovation in cancer research and product development, and to enhance access to evidence-based prevention programs [15]. All CPRIT-funded research is conducted in Texas and reflects CPRIT's mission to develop and implement the Texas Cancer Plan, attract and expand the state's research capabilities, and create high-quality new jobs in Texas.

CPRIT released the 2018 Texas Cancer Plan in September 2018. The Plan identifies five high-priority areas, each poised to have a significant impact on cancer, and it also reflects changes, progresses, and advances in cancer prevention and control since the last revision in 2012. The 2018 Texas Cancer Plan can be found at http://www.texascance rplan.org.
Texas continues to address cancer through the implementation of the Texas Cancer Plan. Texas death rates from cancer decreased by 7\% between 2009 and 2014 (the latest data available); this translates to nearly 6,800 averted deaths. Advances in research, the implementation of evidence-based interventions, early detection of cancer and better treatments have been instrumental to achieving this decrease. The latest available data (2014-2017) were compared to the 2012 baseline data to evaluate progress toward achieving the goals of the Plan [16]:

- The percentage of youth and adults who smoke declined and $39.9 \%$ of Texans are now covered by a comprehensive smoke-free ordinance.

- HPV vaccination completion rates (now two doses) increased for females and almost doubled for males (14.2-26\%).

- The screening rates have increased for breast and colorectal cancer.

- Breast, colorectal, and lung cancer and melanoma incidence and mortality rates have declined.

- From 2012 to 2017, the uninsured rates declined from 27 to $16.6 \%$ and Texas had an improved grade for cancer survivor pain and symptom management.

Although progress is being made in addressing the overall burden of cancer in Texas, cancer is the second leading cause of death in Texas, with lung cancer as the leading cause of death from cancer [17]. In 2018, it is estimated that over 121,463 Texans will be told that they have cancer [18]. These individuals, along with their friends and families, will join thousands of other Texans who are fighting for hope and a cure. It is also estimated that in 2018, over 44,713 Texans will lose their lives to cancer-this equates to more than 122 Texans lost each day to the disease. Almost every Texan, whether connected personally or through friends or family members, has been affected by cancer [19].

Some segments of the Texas population are affected by cancer to a greater degree than others. For example, some racial and ethnic groups are more likely than others to have cancer discovered at a later stage, leading to higher mortality rates. Eliminating cancer disparities due to factors such as socioeconomic status (income, education level, insurance coverage, etc.), race, ethnicity, geographic location, and gender is a cross-cutting aim of the Texas Cancer Plan update. These unique issues present challenges as well as opportunities.

TCCCP and CAT will continue to consider such challenges when implementing the Texas Cancer Plan. CCC champions understand that progress is made through innovation, collaboration, and understanding populations being served. Texas will continue its focus on primary prevention, 
early detection, better treatment, and enhanced survivorship. This will be accomplished by:

- Increasing the coordination, integration, and implementation of cancer activities in the state as identified in the Texas Cancer Plan (Plan).

- Increasing the use of the Plan.

- Developing a data-driven process for prioritizing the Plan.

- Enhancing implementation of the Plan at the local level through a network of cancer stakeholders at the community level.

\section{Conclusion}

The six examples above illustrate how CCC coalitions and programs have leveraged existing resources and created new opportunities that have contributed to CCC movement over the past 20 years. Their success can be measured in cancers prevented, lives saved due to finding cancer early, and cancer survivors living longer and healthier lives in communities that are built to support long-lasting changes. While much progress has been made, there is much to do-the articles in this Special Issue offer ideas for current and future action that all of us can take to help conquer cancer.

\section{Special Issue Editorial Committee Members}

Leslie Given, Guest Editor, Strategic Health Concepts

Margaret Farrell, National Cancer Institute

Lorrie Graaf, American Cancer Society (currently with National Association of Chronic Disease Directors)

Nikki Hayes, Centers for Disease Control and Prevention Karin Hohman, Strategic Health Concepts

Nina Miller, American College of Surgeons Commission on Cancer

Behnoosh Momin, Centers for Disease Control and Prevention

Sarah Shafir, American Cancer Society

Aubrey Van Kirk Villalobos, George Washington University Cancer Center

Cynthia Vinson, National Cancer Institute

Acknowledgments The Editorial Committee would like to recognize and thank the following individuals for their expertise and review of the articles in this Special Issue: Brad Belnap, Utah Department of Health; Polly Hager, Michigan Department of Health and Human Services; Krystal Morwood, Colorado Department of Public Health and Environment; and Keylee Wright, Indiana State Department of Health. The guest editor of this Special Issue would like to thank the Editorial Committee members for their work and guidance in developing this
Special Issue and for their leadership in moving the comprehensive cancer control approach forward in the US.

\section{References}

1. American Cancer Society. The history of Cancer: Cancer in the twenty-first century. https://www.cancer.org/cancer/cancer-basics/ history-of-cancer/twenty-first-century-and-beyond.html. Accessed 25 Sept 2018

2. Colorado Central Cancer Registry (2018) Colorado Department of Public Health and Environment, Denver. https://www.colorado. gov/pacific/cdphe/cancerregistry. Accessed 14 Nov 2018

3. Miller SE, Hager P, Lopez K, Salinas J, Shepherd WL (2009) The past, present, and future of comprehensive cancer control from the state and tribal perspective. Prev Chronic Dis 6(4):A112. http:// www.cdc.gov/pcd/issues/2009/oct/09_0063.htm. Accessed 25 Apr 2018

4. Accomplishments (2018) Michigan Cancer Consortium, Okemos. http://www.michigancancer.org/AboutTheMCC/Accomplish ments.html. Accessed 24 July 2018

5. Michigan's cancer plan (2018) Michigan Cancer Consortium, Okemos. http://www.michigancancer.org/CancerPlan/Compr ehensiveCancerControlPlan-2016-2020.html. Accessed 24 July 2018

6. Cancer plan dashboard (2018) Michigan Cancer Consortium, Okemos. http://www.michigancancer.org/CancerPlan/Dashboard. html. Accessed 24 July 2018

7. Wilson T, Shamo F, Boynton K, Kiley J (2012) The impact of Michigan's Dr. Ron Davis smoke-free air law on levels of cotinine, tobacco-specific lung carcinogen and severity of self-reported respiratory symptoms among non-smoking bar employees. Tob Control. https://doi.org/10.1136/tobaccocontrol-2011-050328

8. Accomplishments (2018) Tobacco Cessation Collaborative. Michigan Cancer Consortium, Okemos. http://www.michigancancer. org/PDFs/Spotlight/MichTobQuitlineOpp-2014/SmokingCessatio nTalkingPointsandResources2014.pdf. Accessed 25 Apr 2018

9. MCC Board of Directors Meetings (2018) Michigan Cancer Consortium, Okemos. http://www.michigancancer.org/PDFs/About TheMCC/MCCMeetings/BoDMtg/MCCMtgMinutes/2018/03281 8/MichiganSurvivorshipCollaborativeUpdate.pdf. Accessed 24 July 2018

10. Reports, Publications and Media Materials (2018) Northwest Portland Area Indian Health Board, Portland. http://216.243.141.9/ programs/project/nwtccp_reports_publications_media_materials. Accessed 14 Nov 2018

11. Data/Statistics (2018) Northwest Portland Area Indian Health Board, Portland. http://216.243.141.9/programs/project/nwtcc p_data_statistics. Accessed 14 Nov 2018

12. Archives (2018) Northwest Portland Area Indian Health Board, Portland. http://216.243.141.9/programs/archives. Accessed 14 Nov 2018

13. Mission (2018) Cancer Alliance of Texas. https://www.dshs.texas .gov/tcccp/CancerAllianceofTexas.shtm. Accessed 20 Apr 2018

14. An Inventory of Cancer Council Records at the Texas State Archives (1984-2008) Minutes and agenda, Texas Cancer Council records. Archives and Information Services Division, Texas State Library and Archives Commission. https://legacy.lib.utexas.edu/ taro/tslac/30028/tsl-30028.html. Accessed 20 Apr 2018

15. Overview. Cancer Prevention and Research Institute of Texas (CPRIT). http://www.cprit.state.tx.us/about-cprit/. Accessed 20 Apr 2018

16. Cancer in Texas (2017) Texas cancer registry, Texas Department of State Health Services. https://www.dshs.texas.gov/legislativ 
e/2017-Reports/2017-Texas-Cancer-Report.pdf. Accessed 20 Apr 2018

17. Texas Department of State Health Services. Texas health data. http://healthdata.dshs.texas.gov/HealthFactsProfiles. Accessed 20 Apr 2017

18. Texas Department of State Health Services, Center for Health Statistics. Leading causes of death by Texas County of Residence, 2015. Source: Texas Death Certificates. 20 Apr 2018
19. Cancer Epidemiology and Surveillance Branch, Texas Department of State Health Services. Texas cancer registry. https://www.dshs. texas.gov/tcr/. Accessed 20 Apr 2018 